\title{
Is there an unmet pharmacological need in chronic lower back pain?
}

\author{
Jan M. Keppel Hesselink* \\ Professor of molecular pharmacology, consultant drug development, Institute Neuropathic Pain, the Netherlands
}

\begin{abstract}
Lower Back Pain (LBP) is a heterogeneous indication based on many different etiopathogeneses. Placebo response and natural course in LBP are complicating the outcome of drug trials. Non-drug treatments are effective and recommended as first choice in most guidelines. Pharmacological treatments are thus not first choice for the treatment of LBP. Expensive new drugs therefore are discouraged by opinion leaders and by the field itself. Drug effects in general are seen as only moderate to weak, and are often complicated by troublesome side effect issues. LBP, although a huge market, is segmented in many sub-indications, such as arthritic induced pain, sciatic pain, lumbar stenosis and Chronic Discogenic Low Back Pain. Drug development of New Chemical Entities (NCE's) in indications linked to LBP remains a risky business, given the situation above. Most therapies under evaluation for LBP are non-pharmacological, such as acupuncture, progressive relaxation, electromyography biofeedback, low-level laser therapy, operant therapy and cognitive-behavioral therapy. There is no consensus on what are currently promising NCE's in the pipeline (apart from what the companies themselves communicate). It seems that there is no unmet pharmacological need in this segment of the chronic pain market.
\end{abstract}

\section{Introduction}

Low back pain (LBP) is one of the most common health problems, with a lifetime prevalence of $80-85 \%$. Low back pain (LBP) is a common source of pain and disability, but the pathogenesis, etiology and pathophysiology of LBP are quite diverse. Recently the chairman of the American College of Physicians (ACP) added an interesting comment to their guidelines for the noninvasive treatment of nonradicular subacute, acute, and chronic low back pain in primary care, and he stipulated:

"For treatment of chronic low back pain, clinicians should select therapies that have the fewest harms and lowest costs because there were no clear comparative advantages for most treatments compared with one another. Clinicians should avoid prescribing costly therapies; those with substantial potential harms, such as long-term opioids (which can be associated with addiction and accidental overdose); and pharmacologic therapies that were not shown to be effective, such as tricyclic antidepressants and selective serotonin reuptake inhibitors [1]."

While no therapy was clearly better than any other, new evidence supports especially non-pharmacological treatments such as mindfulness-based stress reduction and tai chi in chronic low back pain and acupuncture in acute low back pain. Therefore, the recommendation for the treatment of chronic LBP is to first consider nondrug therapy, such as exercise, multidisciplinary rehabilitation, acupuncture, mindfulness-based stress reduction, tai chi, yoga, motor control exercise, progressive relaxation, electromyography biofeedback, low-level laser therapy, operant therapy, cognitivebehavioral therapy, or spinal manipulation before considering the next step, pharmacological active treatments. If such therapy is selected, NSAIDs are in general regarded as first-line pharmacotherapy and second-line choices are tramadol or duloxetine, according to most guidelines. For patients suffering from LBP who seek medical care, rapid improvements in pain, disability and return to work are seen in the first month.

\section{Marginal efficacy of main interventions}

There is only little evidence on the cost effectiveness of different LBP pharmacological treatments, although many therapies are available. MRI findings as effect modifiers could not be identified in LBP and sciatica and thus differentiation between these two main populations in LBP remain difficult [2]. This also has negative implications for clinical trials in this field, especially since the pathogenesis of LBP and sciatica are quite different from each other. While chronic LBP mostly is based on arthrosis, at least in the elderly population, sciatic pain is based on chronic nerve compression. Arthrosis pains respond favorably to NSAIDs, while nerve compression leads to neuropathic pain and is not responsive to NSAIDs [3]. This underlines the above discussed difficulty in evaluating pharmacological treatment in LBP, as sciatic pain cannot be differentiated based on MRI findings from non-nerve compression pain syndromes.

Clinicians' recommendations for what therapy to select as a first step treatment in LBP varies considerably and there is quite some uncertainty regarding the respective value of such treatments and interventions. A recent Cochrane analysis showed that 6 out of 13 included RCTs supported NSAIDs as more effective than placebo regarding pain intensity, implying that 7 out of 13 studies were negative [4].

Disease-modifying antirheumatic drugs (DMARTS) are also not recommended for LBP [5]. Opiates are as weak in efficacy compared to NSAIDs [6]. Analgesic and centrally acting muscle relaxant such as eperisone has been in use for the treatment of low back pain (LBP)

Correspondence to: Jan M. Keppel Hesselink, professor of molecular pharmacology, consultant drug development, Institute Neuropathic Pain, the Netherlands, E-mail: jan@neuropathie.nu

Key words: lower Back Pain, pharmacologic therapies

Received: june 02, 2017; Accepted: June 28, 2017; Published: June 30, 2017 
without clear effects [7]. Paracetamol does not produce better outcomes than placebo for people with acute LBP, and it is uncertain if it has any effect on chronic LBP [8]. The efficacy of intra-articular facet joint injections for LBP is also questionable [9].

Due to the substantial variability in etiology and pathogenesis different pharmacotherapeutic interventions are linked to certain (sub-)indications in the field of LBP only, and thus cover only a part of the entire LBP spectrum. For instance, ankylosing spondylitis is quite a different cause for LBP compared to fibromyalgia and post-discotomy pains or spinal stenosis, and 'one size fits all' is clearly not indicated.

Although the field is quite skeptical about the size of drug effects in LBP, there are quite some companies developing new drugs for LBP among which [10]: Adynxx, Inc., Aestus Therapeutics, Inc., Array BioPharma Inc., Astellas Pharma Inc., Axsome Therapeutics, Inc., Egalet Corporation, Frontier Biotechnologies Co., Ltd, Gador S.A., Grunenthal GmbH, Immune Pharmaceuticals Inc., Kineta, Inc., MEDRx Co., Ltd., Pfizer Inc., Regeneron Pharmaceuticals, Inc. and Stayble Therapeutics AB. We will review briefly 3 new approaches in the treatment of LBP.

\section{Example of pharmacotherapy for LBP in research or de- velopment phase}

The first example is still an early lead, in research phase: the compound AYX2 from Adynxx, Inc. This compound, according to the company, is designed to produce a robust and long-lasting suppression of chronic pain in response to inflammatory or neuropathic insults. The target profile of AYX2 is based on the company's statement that the compound needs to suppress the maintenance of chronic pain without side-effects and abuse potential. AYX2 functions as a so called a transcription factor decoy [11], and is designed for the treatment of chronic pain following a single or limited number of intrathecal administrations. Transcription factor decoys function as inhibitors of transcription factor activity. Such decoys were tested and led to the inhibition of Kruppel-like transcription factors (KLF), resulting in a reduction of mechanical hypersensitivity in animal models [12]. The company's website communicates that AYX2 is specifically designed to target multiple transcription factors that sustain chronic pain due to inflammatory and/or neuropathic insult. By switching off the neuronal mechanisms that maintain pain, the company hopes that AYX2 can produce long-lasting suppression of multiple chronic pain etiologies. Whether such hope is realistic in LBP remains to be seen, given the complexities of the pathogenesis of LBP, we discussed above.

A second example is the AB001 topical patch of Frontier Biotechnologies Inc. containing a non-disclosed NSAID in development for LBP: AB001 is designed as a topical patch product for the treatment of acute and chronic muscle and joint pain and inflammation. It's a patent-protected and non-hydrogel based matrix formulation for enhanced skin penetration and tissue permeability, both with anti-inflammation and analgesic potency. The patch is said to be easily applied to all skin types and easy to use; the patent protected matrix system is only $200 \mathrm{~mm}$ thick. A randomized, doubleblind, placebo-controlled phase II study of the safety and efficacy of this patch in 146 chronic LBP patients was successfully completed in 2016. Pain scores were $30 \mathrm{~mm}$ or greater on the visual analog scale (VAS) at entry. Patients were randomized in a 1:1 ratio to AB001 patches or placebo patches. Two patches were given topically once daily for 14 days. Frontier Biotechnologies Inc. reported the results in 2016: AB001 met primary endpoint at week-2, demonstrating statistically significant $(\mathrm{p}=0.023)$ and clinically meaningful pain relief against placebo. However, we could not identify the results in peer reviewed journals, and in clinicaltrials.gov we could not identify new phase III studies conducted with the patch.

A third example is drug repositioning of old drugs in the field of Discogenic Chronic Low Back Pain (cLBP). For instance, via a minimally invasive, single injection of a not disclosed drug into the disc, as developed by Stayble therapeutics, known under the code name STA363. In clinicaltrials.gov the compound was identified as xylometazoline, the company did not disclose the drugname yet, and a phase IIa study in 15 patients is planned to start in 2017. The technology is based on a use patent for the use of an existing drug substance for the treatment of cLBP. The treatment according to the company is presumed to be effective within 4-12 weeks and a single treatment will be enough, while the treatment does not require extensive rehabilitation. The compound is said to have been used for decades which might allowing a relatively fast, inexpensive and lowrisk route to market, as documented for most repositioned drugs.

\section{Overview of therapies under evaluation}

In ClinTrials.gov (explored in April 2017) there are 1380 studies described for LBP, from a number of NSAIDs (e.g. etoricoxib), fentanyl, oxycodone, tramadol to MOABs such as tanezumab ((RN624, Pfizer) and the anti-nerve growth factor monoclonal antibody JNJ-42160443 from Johnson \& Johnson, duloxetine, Botulinum Toxin A and fish oil to topical treatments such as the AB001 topical patches and ketoprofen gel.

Most studies however seem to evaluate non-pharmacological treatments: Yoga, Qigong, craniosacral, Feldenkrais, TENS, biofeedback McKensie, acupuncture, spinal manipulative therapy, up to Autologous Platelet Rich Plasma etc. And recently reviewed evidence suggested that combined physical exercise and psychological treatments (CBT and risk stratification), provision of information and manual therapy (chiefly spinal manipulation and acupuncture) are cost-effective options for LBP [13].

\section{Conclusion}

LBP is not a unity of diagnosis, it is a heterogeneous collection of disorders, and development of pharmacological interventions will need to be focused in sub-segments of this huge market. Segments such as sciatic pain after a lumbar disk hernia, pain due to lumbar stenosis (syndrome of Verbiest) and Chronic Discogenic Low Back Pain are all characterized by a differentiated etiopathogenesis, and different pharmacological interventions will have different spectra of activity in these indications. There is indeed an unmet need in a number of these sub segments, but one can detect a general consensus that such complaints do not respond very favorably to pharmacological interventions in general. It would be interesting to explore the number of negative trials in this segment: it will be significant. Most complaints die away in months (including natural regression to the mean). This clearly explains the complexities (diagnosis, coherent population, in and exclusion criteria, endpoints) and complications one needs to conquer in setting up clinical trials in this indication. For the time being the market seems not motivated for high priced NCE's entering the various segments of chronic LBP, and therefore it seems that there is no unmet need for pharmacological interventions in this segment of the pain market. 


\section{References}

1. New ACP Guidelines for Nonradicular Low Back Pain. http://www.medscape.com/ viewarticle/875737

2. Steffens D, Hancock MJ, Pereira LS, Kent PM, et al. (2016) Do MRI findings identify patients with low back pain or sciatica who respond better to particular interventions? A systematic review. Eur Spine J 25: 1170-1187. [Crossref]

3. Rasmussen-Barr E, Held U, Grooten WJ, Roelofs PD, Koes BW, et al. (2016) Nonsteroidal anti-inflammatory drugs for sciatica. Cochrane Database Syst Rev 10: CD012382. [Crossref]

4. Enthoven WT, Roelofs PD, Deyo RA, van Tulder MW, Koes BW (2016) Non-steroida anti-inflammatory drugs for chronic low back pain. Cochrane Database Syst Rev 2: CD012087. [Crossref]

5. Malik KM, Nelson A, Benzon H (2016) Disease-modifying Antirheumatic Drugs for the Treatment of Low Back Pain: A Systematic Review of the Literature. Pain Pract 16: 629-641. [Crossref]

6. Berthelot JM, Darrieutort-Lafitte C, Le Goff B, Maugars Y (2015) Strong opioids for noncancer pain due to musculoskeletal diseases: Not more effective than acetaminophen or NSAIDs. Joint Bone Spine 82: 397-401. [Crossref]
7. Bavage S, Durg S, Ali Kareem S, Dhadde SB (2016) Clinical efficacy and safety of eperisone for low back pain: A systematic literature review. Pharmacol Rep 68: 903912. [Crossref]

8. Saragiotto BT, Machado GC, Ferreira ML, Pinheiro MB, Abdel Shaheed C, et al. (2016) Paracetamol for low back pain. Cochrane Database Syst Rev : CD012230. [Crossref]

9. Vekaria R, Bhatt R, Ellard DR, Henschke N, Underwood M, et al. (2016) Intra-articular facet joint injections for low back pain: a systematic review. Eur Spine J 25: 1266-1281. [Crossref]

10. http://www.researchandmarkets.com/reports/3743752/low-back-pain-pipelinereview-h1-2016.pdf

11. Mann MJ (2005) Transcription factor decoys: a new model for disease intervention. Ann N Y Acad Sci 1058: 128-139. [Crossref]

12. Mamet J, Harris S, Klukinov M, Yeomans DC, Donahue RR, et al. (2017) Pharmacology, pharmacokinetics, and metabolism of the DNA-decoy AYX1 for the prevention of acute and chronic post-surgical pain. Mol Pain 13: 1744806917703112. [Crossref]

13. Andronis L, Kinghorn P, Qiao S, Whitehurst DG, Durrell S, et al. (2017) Cost-Effectivenes of Non-Invasive and Non-Pharmacological Interventions for Low Back Pain: a Systematic Literature Review. Appl Health Econ Health Policy 15: 173-201. [Crossref]

Copyright: (C2017 Hesselink JMK. This is an open-access article distributed under the terms of the Creative Commons Attribution License, which permits unrestricted use, distribution, and reproduction in any medium, provided the original author and source are credited. 